\title{
A Combi-Stroop Test for Measuring Food-Related Attentional Bias
}

\author{
Javad Salehi Fadardi and Masoud Moghaddaszadeh Bazzaz \\ Ferdowsi University of Mashhad
}

\begin{abstract}
There are methodological complexities with the supraliminal-lexical versions of the modified versions of the Stroop tests that could be responsible for inconsistencies across the literature (Field \& Cox, 2008). We tested whether a combination of subliminal-pictorial and classic Stroop tests can differentiate between dieters' and nondieters' food attentional bias (FAB). Participants were dieters $(n=30)$ and nondieters $(n=32)$ who were tested $3 \mathrm{hr}$ after having a meal. Each picture from among 24 high-calorie and 24 low-calorie food pictures was presented for $32 \mathrm{~ms}$ before the appearance of a congruent or an incongruent color word, in response to which participants were required to manually report, via a tagged keyboard, the correct color of the word as quickly and accurately as possible. Color-naming latencies and interference scores were calculated. Dieters showed the highest reaction times to incongruent color words following high-calorie food pictures; overall, dieters showed significantly higher FABs than nondieters. The Combi-Stroop test has differential validity. Moreover, findings suggest that FAB can result from early allocation of dieters' attention to food-related stimuli.
\end{abstract}

Keywords: attentional bias, diet, obesity, overweight, emotional Stroop test, subliminal, supraliminal

Obesity and overweight are associated with many adverse health consequences (for review, see Bean, Stewart, \& Olbrisch, 2008); hence, it has become the focus of many researchers from various disciplines. It has been estimated that more than 1 billion people in the world suffer from overweight, among whom 400,000,000 are classified as obese, that is, their body mass index is higher than 30 (World Health Organization, 2010). In Iran, it was estimated that, in $2005,48.5 \%$ males and $57.8 \%$ females were overweight. It has been estimated that the rate will remain relatively unchanged for males, but for females, it will reach $62.5 \%$ in 2015 (World Health Organization, 2010).

There are several methods of treatment for obesity and overweight, among which dieting is the most popular. Dieters generally try to reduce their weight through limiting their calorie intake. However, few dieters maintain their diet; most resume their old eating habits. The question is why they fail to do so despite their conscious awareness about the harms of unhealthy eating and their intention to change their eating habits. Could dieting increase dieters' craving for forbidden food? Are there any aspects of eating habits that may be out of the conscious control of the dieters?

To answer questions addressing uncontrollable facets of eating behaviors, some cognitive psychologists have con-

This article was published Online First May 30, 2011.

Javad Salehi Fadardi and Masoud Moghaddaszadeh Bazzaz, Department of Psychology, Ferdowsi University of Mashhad, Mashhad, Iran.

We thank Steven Hosier, who kindly provided useful comments on a version of this article.

Correspondence concerning this article should be addressed to Javad Salehi Fadardi, Department of Psychology, Ferdowsi University of Mashhad, Mashhad, Iran. E-mail: j.s.fadardi@um.ac.ir centrated on implicit cognitions and attentional processes that may underlie the behavior (e.g., Papies, Stroebe, \& Aarts, 2009; Stroebe, Mensink, Aarts, Schut, \& Kruglanski, 2008). In short, the common finding of these studies is that automatic attentional processes play an important role in the continuation of or relapsing to old eating habits. For example, it has been argued (e.g., Calitri, Pothos, Tapper, Brunstrom, \& Rogers, 2010) that food attentional bias (FAB) may distort cognitive processes that are responsible for decisions to eat. Although foods do not cause physical dependence as alcohol or some drugs do, their strong incentive values can render them just as addictive (Tapper, Pothos, Fadardi, \& Ziori, 2008). It seems that information processes responsible for consuming behaviors in dieters and food restrainers are similar to those observed in alcohol and drug abusers (Cox, Fadardi, \& Pothos, 2006). One similarity between food and alcohol, for example, is that pleasant effects of both are mediated by mu-opiate receptors in the ventral striatum of the brain, which is an important part of the brain's reward system (Heinz et al., 2005). Some researchers (e.g., Cannon \& Bseikri, 2004; Robinson \& Berridge, 2001) have argued that the main reason for some chemicals to become addictive is that, similar to natural incentives (e.g., food), they activate the brain's dopaminergic system. Cox, Fadardi, and Pothos (2006) proposed the process through which any type of incentive (e.g., food, substances) can sensitize the person's attentional system. In their account, certain foods or chemicals may find extreme incentive value (e.g., it helps a person reduce his or her stress or feel euphoric); therefore, eating a given food may become a continuous goal for the person. A goal is associated with an internal motivational state termed current concern, which is an active, goal-lurking agenda that leads the person toward achieving his or her goal. By repeating a given rewarding behavior, the person's attentional sensitiv- 
ity for goal-related stimuli changes to a hypersensitivity state, which is more or less out of the person's conscious control. For instance, substance abusers show an attentional bias for substance-related stimuli; that is, they are hypersensitive to any cues related to various aspects of substance use, and being exposed to these cues may instigate automatic behaviors that may cause them to go back on their word - that is, through searching, obtaining, and using the substance (e.g., Cox, Fadardi, \& Pothos, 2006; Field \& Cox, 2008).

Evidence from clinical samples, such as bulimics and anorexics, supports the importance of attentional processes in pathological eating behaviors (e.g., Dobson \& Dozois, 2004; Jones-Chesters, Monsell, \& Cooper, 1998). However, there has not been much research with nonclinical samples, such as obese and overweight people, dieters, or restrained eaters (Tapper et al., 2008). However, findings with nonclinical samples are inconsistent. For example, Green and Rogers (1993) and Perpina, Hemsley, Treasure, and de Silva (1993) found that restrained or dieting women with no obvious clinical problems showed higher FAB on a modified Stroop test than controls. However, the results of studies conducted by Ben-Tovim and Walker (1991) and Long, Hinton, and Gillespie (1994) suggest that mere current concerns with food and eating are not associated with increases in FAB (compared with controls), but a concurrent clinical diagnosis for eating-related disorders is a necessary condition to observe a statistically significant difference among the two groups.

In a meta-analysis of 27 food-related Stroop studies, Johansson, Ghaderi, and Andersson (2005) concluded that there is no consistent evidence in support of the assumption that restrained eaters should show increased FAB than controls. However, the authors suggested that the lack of support may simply result from some methodological issue or the scarce number of studies on dieters compared with clinical samples. Accordingly, considering the growing numbers of dieters and restrained eaters, it is necessary to study various aspects of the problem, including any relevant cognitive and attentional processes.

A commonly used paradigm to study FAB is a modified version of the emotional Stroop test (Williams, Mathews, \& MacLeod, 1996); the test has clinical applications (Gallagher-Duffy, MacKay, Duffy, Sullivan-Thomas, \& Peterson-Badali, 2009) and it can also be used to scrutinize attentional processes (e.g., strategic vs. automatic) involved in hypersensitivity for emotionally salient stimuli (e.g., Cisler, Bacon, \& Williams, 2009; Johansson et al., 2005). An emotional Stroop test usually comprises two categories of stimuli (commonly words): a salient, current concernrelated category and a control, emotionally neutral category (see Cox, Fadardi, \& Pothos, 2006). The stimuli have to be matched in terms of various relevant dimensions, such as lexical dimensions, if words are used; thereby, differences should be minimized across the two categories of stimuli except for their relevance or irrelevance to the target emotional or current concern category. In the case of words, all stimuli are presented in various colors (e.g., red, blue, green, yellow), and the task for the participant is to ignore the meaning of the words and respond as accurately and quickly as possible to their color. The rationale behind the test is that having a current concern for the meaning of a word distracts the person, causing slower reaction times to the salient category than to the neutral category of the stimuli. The difference between the person's mean reaction times to the two categories is a measure of one's distractibility or attentional bias for the salient stimuli (Bruce \& Jones, 2004; Williams et al., 1996).

\section{Lexical Versus Pictorial Stimuli}

In many Stroop studies on attentional bias, words have been used as salient, distracting stimuli. However, many researchers (e.g., Bruce \& Jones, 2004; Johansson et al., 2005) believe that pictorial stimuli are more potent in assessing attentional bias than words because they are more representative of the world. Papies et al. (2009) believe that pictorial stimuli contribute to a more accurate assessment of automatic processing in the assessment of FAB than words that initially involve lexical processes. In a study with clinically diagnosed patients with eating disorders, Walker, Ben-Tovim, Paddick, and McNamara (1995) used pictures that were related to body image; they found that anorexic and bulimics took longer to respond to body pictures than to neutral pictures, whereas the controls did not show a difference in their responses to the two types of stimuli.

However, in our review of emotional, pictorial versions of the Stroop test, we realized that most studies used black-andwhite or mono-colored photos (e.g., Bruce \& Jones, 2004; Constantine, McNally, \& Horning, 2001; Harrison, Sullivan, Tchanturia, \& Treasure, 2010; Walker et al., 1995). Searching PubMed at the time of writing this article, we found that only four of 56 eating-related Stroop studies used pictorial stimuli, which where mono-colored pictures. However, our experience from the real world indicates that advertisements and objects related to eating and food normally present a full range of colors and are more or less close representatives of the actual things (e.g., ads for soft drinks or chocolates). Many dieters report being tempted by seeing such realistic photos, which may finally cause them to go back to their old eating habits. Therefore, to further simulate the actual conditions, we used colorful food images as the salient stimuli in our Stroop test.

\section{Supraliminal Versus Subliminal Versions of the Stroop Test}

In psychology of sensation and perception, subliminal stimuli are those that are presented below the conscious perceptual threshold of an individual. It has been documented that stimuli that are presented just below individuals' perceptual thresholds may produce some moderating effects on decision-making or behavioral processes (e.g., de Gardelle, Charles, \& Kouider, 2011; Hofmann, De Houwer, Perugini, Baeyens, \& Crombez, 2010; Van den Bussche, Van den Noortgate, \& Reynvoet, 2009). In fact, recent evidence from neurological studies that have applied functional magnetic resonance imaging techniques suggests that 
responses primed by subliminal and supraliminal stimuli are modulated by different brain structures (e.g., Bijsterbosch et al., 2011).

Some researchers have studied attentional bias in respect to the time of stimuli presentation, which can lead to subliminal or supraliminal versions of attentional tasks (e.g., Arntz, Appels, \& Sieswerda, 2000; Lundh, Wikstrom, Westerlund, \& Ost, 1999; Mogg, Bradley, Williams, \& Mathews, 1993; Stroebe et al., 2008). Mogg et al. (1993) and Stroebe et al. (2008) noted that subliminal presentation of the stimuli can help neutralize the effect of strategic controlling processes.

Moreover, as Kemps and Tiggemann (2009) have argued, supraliminal presentation of stimuli makes it hard to distinguish between two potential sources of attentional bias: one unconscious and preattentive; the other, effortful and postconscious. Such differentiations have important implications for both theory and treatment of attentional bias for psychopathologically related stimuli.

Houben, Roefs, and Jansen (2010) argued that eating can be guided through two types of cognitive processes: (a) reflective or explicit and (b) impulsive or implicit. The explicit processes include volitional decisions, reasoning, and personal goals and norms that are normally characterized with the person's efforts; they are slow and obvious and reliant on cognitive resources. It seems that the negative reactions of some dieters to high-calorie food are due to these reflective, explicit processes. Impulsive, implicit processes, however, are automatic, hidden, and fast, and they do not require much effort; these explain dieters' tendencies and positive responses toward high-calorie food. Therefore, because of their nature, it is difficult to control implicit cognitive processes because they occur readily, involuntarily, and are out of the person's conscious awareness. To conclude, the more we know about these processes, the better we understand the nature of many dysfunctional behaviors such as overeating - and why people choose to do them despite their conscious awareness of the harm that they may cause (Wiers, Teachman, \& De Houwer, 2007).

\section{Initial Versus Late Stages of Processing}

Papies et al. (2009) believe that one advantage of measuring individuals' implicit, automatic cognitions is that these measurements are less vulnerable to respondents' conscious efforts to disguise their actual ways of thinking and reacting to the salient stimuli, hence reducing the risk of regression toward the mean. However, it seems that more subtle distractions for emotionally salient stimuli may be hard to observe using implicit measures of attentional bias that mainly focus on the final (i.e., response level) instead of the initial levels of processing of the stimuli. That is, any distraction for emotionally salient stimuli may be observable for two reasons: (a) It starts immediately after being exposed to the salient stimuli, and (b) it continues to affect one's responses provided that conscious, compensatory strategies have not been used. Evidence suggests that it is possible to distinguish between the initial- and responselevel sources of attentional bias. Stimulus onset asynchrony
(SOA; e.g., McNamara, 2005) refers to the amount of time between the presentation of a prime (e.g., pictures in a dot-probe task) and the presentation of a target (e.g., the dot in a dot-probe task) that allows differentiating the two levels of processing. Evidence suggests that when the SOA is very short (e.g., $50 \mathrm{~ms}$ ), it is less likely that respondents can use their response strategies to overcome their initial distraction for emotionally salient stimuli (e.g., Vollstadt-Klein, Loeber, von der Goltz, Mann, \& Kiefer, 2009). In his review of studies varying the SOA, MacLeod (1991) concluded that SOAs less than $100 \mathrm{~ms}$ can increase the size of the interference. In summary, subliminal presentation of the stimuli as well as short SOAs are techniques used to increase the size of interference that most probably can be attributed to the initial stages of processing.

However, when searching for subliminal Stroop and attentional bias in PubMed, we found only 10 relevant studies, of which seven (i.e., Franken, Kroon, Wiers, \& Jansen, 2000; Jansen, Huygens, \& Tenney, 1998; Mogg \& Bradley, 2002; Paunovi, Lundh, \& Ost, 2002; Sackville, Schotte, Touyz, Griffiths, \& Beumont, 1998; Thorpe \& Salkovskis, 1997; Zetteler, Stollery, Weinstein, \& Lingford-Hughes, 2006) showed that subliminal versions of the emotional Stroop tests failed to show the expected interference, whereas the supraliminal versions did. Nonetheless, other studies (i.e., Afzal, Potokar, Probert, \& Munafo, 2006; Bakvis et al., 2009; Edwards, Burt, \& Lipp, 2006; van der Made et al., 2009) reported attentional bias for the salient stimuli at a preattentive level.

The goal of the current study was to develop a new emotional Stroop test that selectively combines techniques from preattentive presentation of the stimuli and classic Stroop test to reduce participants' potential use of conscious strategies that may attenuate an initially provoked interference.

\section{Method}

\section{Participants}

Dieters $(n=30$; mean age $=25$ years, $S D=3.2 ; 100 \%$ women) were among patients referred to a dieting clinic in Mashhad to lose weight. They did not meet any of the clinical eating disorders criteria (e.g., anorexia nervosa). Control participants were students at Ferdowsi University of Mashhad $(n=32$; mean age $=22$ years; $S D=2.5 ; 100 \%$ women), who responded to the experimenter's invitation to voluntarily participate in the study. Participants in the control group were not on a diet.

\section{The Combi-Stroop Test}

The Combi-Stroop test was developed for this study for the first time. In general, we designed a Combi-Stroop test as one that (a) includes pictures of a salient (e.g., target) or an alternative (e.g., control) category-the pictures can be presented at the subliminal or supraliminal level; (b) congruent and incongruent color words that follow each picture in an equal proportion; and (c) an SOA that can vary. 


\section{Stimuli and Apparatus}

In the current study, 24 photos of soft drinks (e.g., Iranian cola brands) and high-calorie food (e.g., chocolate, french fries, pizza) and 24 photos of natural drinks (e.g., water, yogurt drink) and low-calorie food (e.g., celery, orange, vegetable salad) were used. Photoshop software was used to trim the pictures; on average, all pictures had $300 \times 300$ pixels, with no additional details in a white background square; however, the whole square (including the foreground and the white background) was depicted against a black background and presented for $32 \mathrm{~ms}$ during the test.

Within each picture category, each picture was followed once by a congruent color word (e.g., green in green) and once by an incongruent color word (e.g., red in green). The color words were blue, green, yellow, and red, again, presented against a black background. The interval between the photos and words was set at zero. There was, however, a fixation cross that followed each color word upon the participant's response or if $2 \mathrm{~s}$ had elapsed. Therefore, each picture and word made a pair; between each pair of stimuli there was a white fixation cross for $500 \mathrm{~ms}$ on a black background. In summary, the test comprised 48 pictures and 48 color words.

SuperLab Pro2.0 SKD (Cedrus, 1999) was used to develop and present the Combi-Stroop test. A PC was used for running the program on a standard 15 -in. monitor that was located about $40 \mathrm{~cm}$ away from the participant's eyes. A standard keyboard was used as the input device on which four keys were marked as response keys (i.e., ? for red, the less than symbol $[<]$ for yellow, $C$ for green, $Z$ for blue). The computer recorded reaction times (RTs) to each stimulus as well as correct and error (i.e., wrong and late) responses.

\section{Procedure}

The study had been announced as one measuring RTs to a series of color words on a computer display. All participants were given an information sheet about the study and were encouraged to participate in the study voluntarily. They signed a consent form prior to their participation, and they were required to refrain from eating for at least $3 \mathrm{hr}$ before the testing session.

The test was administered in a quiet room with normal illumination. Participants were given enough practice with the computer to learn the correct position of each color key and familiarize themselves with the task prior to the main task. The practice trials comprised responses to 70 color patches corresponding to the colors used in the actual test. On the Combi-Stroop test, the task was explained as responding to the color of the color words as accurately and quickly as possible while ignoring the meaning of the words. The testing sessions were conducted in the mornings and afternoons. To ensure that the picture presentation was at the subliminal level, we asked all participants at the end of their test about the content of the pictures that were presented prior to each word; only seven of 59 participants guessed that they had seen pictures related to food. Initially, information collected from all participants was included in the data analyses.

\section{Results}

First, we calculated the mean number of error responses (including mistakes and misses) to each category of words. The mean numbers of errors made by all participants were 0.79 $(S D=1.18)$ on congruent color words following low-calorie food (ConLCF), $1.78(S D=2.68)$ on incongruent color words following low-calorie food (InconLCF), 0.95 ( $S D=1.006$ ) on congruent color words following high-calorie food (ConHCF), and $3.09(S D=2.13)$ on incongruent color words following high-calorie food (InconHCF). On average, participants made 3.2\% errors across the Stroop test. The results of a series of paired $t$ tests showed that participants made more errors (a) on InconLCF than ConLCF, $t(63)=3.004, p<.001$; (b) on InconHCF than ConHCF, $t(63)=8.65, p<.001$; and (c) on InconHCF than InconLCF, $t(63)=6.22, p<.001$. The results of a multivariate analysis of variance (MANOVA) comparing dieters and nondieters on the four types of errors showed no significant effect $(p>.05)$.

Second, we calculated mean correct RTs to each category of words. Mean RTs for one dieter and one nondieter were extraordinary large and considered outliers; hence, they were omitted from further data analysis; this left 29 dieters and 31 nondieters in each group. We calculated two interference scores: one for high-calorie food (HCFint) and the other for low-calorie food (LCFint). To calculate an interference score, we subtracted the mean RTs on congruent color words from the mean RTs on incongruent color words that followed each of the food picture categories. Both interference scores were considered measures of attentional bias for food stimuli in each category (i.e., low and high calorie; see Table 1).

To compare mean RTs to congruent and incongruent stimuli on each of the food categories between dieters and nondieters, we conducted a MANOVA. In the model, RTs for ConLCF, InconLCF, ConHCF, and InconHCF were

Table 1

Means and Standard Deviations for Reaction Times and Interference Scores on Each Stimulus Category for Dieters and Nondieters

\begin{tabular}{lrrrrr}
\hline & \multicolumn{4}{c}{ Group } \\
\cline { 2 - 3 } \multicolumn{1}{c}{ Category } & Mean & $S D$ & & Mondieter \\
\cline { 2 - 3 } \cline { 5 - 6 } \cline { 5 - 6 } Mean & $S D$ \\
\hline Low-calorie foods & & & & \\
RT congruent & 797.24 & 165.98 & & 816.90 & 137.71 \\
RT incongruent & 936.48 & 171.20 & & 949.78 & 149.72 \\
LCFint & 134.57 & 89.65 & & 132.87 & 89.61 \\
High-calorie foods & & & & & \\
RT congruent & 835.71 & 175.25 & & 854.96 & 153.81 \\
RT incongruent & 915.91 & 156.11 & & 855.03 & 128.70 \\
HCFint & 80.20 & 102.78 & & 13.08 & 108.71 \\
\hline
\end{tabular}

Note. $\quad \mathrm{RT}=$ reaction time; HCFint $=$ high-calorie foods interference; LCFint $=$ low-calorie foods interference. 
entered as dependent variables and group (dieters vs. nondieters) as the factor. The Wilks's $\Lambda$ test was not significant, $F(4,55)=2.34, p=.066, \eta^{2}=.145$; moreover, scrutinizing the between-subjects effects revealed no effect for group on either of the dependent variables $(p>.05)$. The results of a series of dependent $t$ tests indicated that both groups showed longer latencies for incongruent stimuli than for congruent stimuli, $t(1,30)=9.00, p=.001$.

To determine whether dieters and nondieters differed from each other in terms of their attentional bias for highversus low-calorie food, we conducted another MANOVA. In the model, HCFint and LCFint were entered as dependent variables and group (dieters vs. nondieters) as the factor. The Wilks's $\Lambda$ test was not significant, $F(2,57)=3.09, p=$ $.053, \eta^{2}=.098$; however, there was a significant effect for group on HCFint, $F(1,58)=6.30, p=.015, \eta^{2}=.098$, with dieters showing greater interference than nondieters (see Table 1).

Next, we omitted from the data the six participants (one already omitted as one of the two outliers) who vaguely recognized the content of the pictorial stimuli and repeated the same data analyses. The results of the new data analyses were similar to those obtained with all participants included. However, the $F$ value and the partial effect size for the significant effect for group on HCFint slightly declined, $F(1,52)=4.86, p=.03, \eta^{2}=.086$. The number of the participants who vaguely recognized the stimuli per each group (two nondieters and four dieters) was not large enough to limit the analyses to them or to test their effect as a moderating variable in the main MANOVA model.

\section{Discussion}

For the first time, we developed and tested the application of a new Combi-Stroop test for the assessment of FAB. We believe that using salient images, instead of salient words, increases the similarity between laboratory conditions and the real world. Moreover, subliminal presentation of the salient stimuli reduces participants' conscious efforts to develop response strategies that may adversely affect their performance on the emotional Stroop test as an implicit measure of attentional bias. Together, we started with the assumption that such a combination would represent an accurate measurement of automatic distractibility for emotionally salient stimuli. Although the present findings support the feasibility of using the Combi-Stroop test in measuring FAB, the relative power of this test compared with traditional word versions of the test awaits future research.

Our findings have a number of implications for the emotional Stroop tests. First, we conclude that increasing the difficulty of the task increases chances to observe interference. Thus, our participants showed greater RTs when they were required to respond to the incongruent category of the words. Responding to the incongruent words that followed forbidden food pictures was even harder for the dieters. The reason for such increments in RTs on the incongruent category is that responding to incongruent words demands more cognitive activity (i.e., to suppress the meaning of the word and to respond to the color of the ink); therefore, any distractor (e.g., forbidden food) requires extra cognitive activities to perform the task, leading to augmented interference. Another reason for dieters showing greater interference could be attributed to the stress associated with the difficulty of responding to incongruent words (especially when primed by pictures of forbidden food). This argument is supported by the significant difference in error rates on InconHCF than InconLCF. In other words, stressful conditions may contribute to larger interference than nonstressful conditions.

The present finding supports both lines of theories that account for the processing sources of attentional bias on the emotional Stroop tests: initial level of processing (i.e., attention capture) and response level of processing (i.e., disengagement). That is, when faced with a salient stimulus on the Combi-Stroop test at a preattentive level, the dieters' attentional resources were automatically drawn toward the content of the stimuli; this is equal to attention capture that happens at the initial level of processing. Moreover, suppressing the meaning of the words in the incongruent category is a task at a conscious, attentive level of processing (i.e., response inhibition), for which one needs to disengage from automatic responding to respond selectively to the color of the ink.

Another finding of the study is related to the fact that dieters showed higher FAB than nondieters. The finding supports a wealth of data that food restraint increases FAB (e.g., Kemps \& Tiggemann, 2009; Tapper et al., 2008). However, this is the first study showing that FAB can result from a hypervigilance for desired food even at a preattentive level. The finding supports the theory of current concern as an explanation for attentional bias for emotionally (and more precisely, motivationally) salient stimuli (see Cox, Fadardi, \& Klinger, 2006). The implication of preattentive FAB is that decisions to break one's vow to diet could also be made at a preattentive level, where there is little resistance against craving or alternative, consciously guided decisions. Such a preconscious cognitive mechanism could be responsible for some dieters' frequent failures. Therefore, some dieters may find it difficult to escape from the yo-yo effect or weight cycling (Atkinson et al., 1994); the effect could be exacerbated in cases of quick, tough, but unreasonable demands to lose weight, a situation that is associated with hypervigilance for cues related to forbidden food that weakens behavioral decision making, especially when under stress. In an analysis of drug addiction, Tiffany (1990) proposed that cues related to a well-established habit can lead to compulsive behaviors that are independent of conscious/declarative knowledge of action-outcome relationships. The strength of cue-elicited habits in the presence of active goals (e.g., eating) also has been documented (e.g., Aarts \& Dijksterhuis, 2000; Orbell \& Verplanken, 2010). However, although very difficult, it seems that a provoked automatic behavior that enters one's consciousness can be subjected to the person's conscious review and control (e.g., Brunstrom, Yates, \& Witcomb, 2004). For example, there is evidence (e.g., Blair, Booth, Lewis, \& Wainwright, 1989; 
Brug, Hospers, \& Kok, 1996) that many dieters and restrained eaters can manage weight loss and maintain it over a long time.

It is difficult to generalize our findings to various versions of the emotional Stroop test (e.g., alcohol, substances, anxiety, depression) in that some use more objective stimuli (e.g., words related to objects), whereas others may use stimuli that are rather subjective (e.g., words related to concepts). Moreover, it is still premature to judge whether cultural diversities may lead to different patterns of results when comparing subliminal versions of emotional Stroop tests. However, we predict that, compared with current versions of the emotional Stroop tests, a Combi-Stroop test will result in fewer differences across members of various cultures because it reduces conscious resources that may be used by participants to develop response strategies (see Williams et al., 1996).

To conclude, it seems that the Combi-Stroop test can be reliably used as a measure of FAB. The present study, however, could be improved by the inclusion of a third category of neutral pictorial stimuli. That way, we could determine whether, compared with controls, dieters are generally distracted for any type of food (i.e., healthy and forbidden) or whether they show greater FAB exclusively for forbidden foods. Finally, we suggest comparing the relative discriminant and predictive validity of the CombiStroop test with its traditional versions. Moreover, the relative rigor of supraliminal versions of the Combi-Stroop, in comparison with its subliminal versions, for detecting FAB awaits future studies.

\section{References}

Aarts, H., \& Dijksterhuis, A. (2000). Habits as knowledge structures: Automaticity in goal-directed behavior. Journal of Personality and Social Psychology, 78, 53-63.

Afzal, M., Potokar, J. P., Probert, C. S., \& Munafo, M. R. (2006). Selective processing of gastrointestinal symptom-related stimuli in irritable bowel syndrome. Psychosomatic Medicine, 68, 758761.

Arntz, A., Appels, C., \& Sieswerda, S. (2000). Hypervigilance in borderline disorder: A test with the emotional Stroop paradigm. Journal of Personality Disorders, 14, 366-373.

Atkinson, R. L., Dietz, W. H., Foreyt, J. P., Goodwin, N. J., Hill, J. O., .. Yanovski, S. Z. (1994). Weight Cycling. JamaJournal of the American Medical Association, 272, 1196-1202.

Bakvis, P., Roelofs, K., Kuyk, J., Edelbroek, P. M., Swinkels, W. A., \& Spinhoven, P. (2009). Trauma, stress, and preconscious threat processing in patients with psychogenic nonepileptic seizures. Epilepsia, 50, 1001-1011.

Bean, M. K., Stewart, K., \& Olbrisch, M. E. (2008). Obesity in America: Implications for clinical and health psychologists. Journal of Clinical Psychology in Medical Settings, 15, 214224.

Ben-Tovim, D. I., \& Walker, M. K. (1991). Hypervigilance in borderline disorders: A test with the emotional Stroop paradigm. International Journal of Eating Disorders, 10, 609-613.

Bijsterbosch, J. D., Lee, K. H., Hunter, M. D., Tsoi, D. T., Lankappa, S., Wilkinson, I. D., . . Woodruff, P. W. (2011). The role of the cerebellum in sub- and supraliminal error correction during sensorimotor synchronization: Evidence from fMRI and TMS. Journal of Cognitive Neuroscience, 23, 1100-1112.
Blair, A. J., Booth, D. A., Lewis, V. J., \& Wainwright, C. J. (1989). The relative success of official and informal weight reduction techniques: Retrospective correlational evidence. Psychology and Health, 3, 195-206.

Bruce, G., \& Jones, B. T. (2004). A pictorial Stroop paradigm reveals an alcohol attentional bias in heavier compared to lighter social drinkers. Journal of Psychopharmacology, 18, 527-533.

Brug, J., Hospers, H. J., \& Kok, G. (1996). Differences in psychosocial factors and fat consumption between stages of change for fat reduction. Psychology and Health, 12, 719-727.

Brunstrom, J. M., Yates, H. M., \& Witcomb, G. L. (2004). Dietary restraint and heightened reactivity to food. Physiology and Behavior, 81, 85-90.

Calitri, R., Pothos, E. M., Tapper, K., Brunstrom, J. M., \& Rogers, P. J. (2010). Cognitive biases to healthy and unhealthy food words predict change in BMI. Obesity, 18, 2282-2287. doi: 10.1038/oby. 2010.83

Cannon, C. M., \& Bseikri, M. R. (2004). Is dopamine required for natural reward? Physiological Behavior, 81, 741-748.

Cedrus-Corporation. (1999). SuperLab Pro SKD (Version 2.1) [Software]. San Pedro, USA: Cedrus-Corporation.

Cisler, J. M., Bacon, A. K., \& Williams, N. L. (2009). Phenomenological characteristics of attentional biases towards threat: A critical review. Cognitive Therapy and Research, 33, 221-234.

Constantine, R., McNally, R. J., \& Horning, C. D. (2001). Snake fear and the pictorial emotional Stroop paradigm. Cognitive Therapy and Research, 25, 757-764.

Cox, W. M., Fadardi, J. S., \& Klinger, E. (2006). Motivational process underlying implicit cognition in addiction. In R. W. Wiers \& A. W. Stacy (Eds.), Handbook of implicit cognition and addiction (pp. 253-266). Thousand Oaks, CA: Sage.

Cox, W. M., Fadardi, J. S., \& Pothos, E. M. (2006). The addictionStroop test: Theoretical considerations and procedural recommendations. Psychological Bulletin, 132, 443-476.

de Gardelle, V., Charles, L., \& Kouider, S. (2011). Perceptual awareness and categorical representation of faces: Evidence from masked priming. Conscious Cognition. Advance online publication. doi:10.1016/j.concog.2011.02.001

Dobson, K. S., \& Dozois, D. J. A. (2004). Attentional biases in eating disorders: A meta-analytic review of Stroop performance. Clinical Psychology Review, 23, 1001-1022.

Edwards, M. S., Burt, J. S., \& Lipp, O. V. (2006). Selective processing of masked and unmasked verbal threat material in anxiety: Influence of an immediate acute stressor. Cognition \& Emotion, 20, 812-835.

Field, M., \& Cox, W. M. (2008). Attentional bias in addictive behaviors: A review of its development, causes, and consequences. Drug and Alcohol Dependence, 97, 1-20.

Franken, I. H., Kroon, L. Y., Wiers, R. W., \& Jansen, A. (2000). Selective cognitive processing of drug cues in heroin dependence. Journal of Psychopharmacology, 14, 395-400.

Gallagher-Duffy, J. G., MacKay, S., Duffy, J., Sullivan-Thomas, M., \& Peterson-Badali, M. (2009). The pictorial fire Stroop: A measure of processing bias for fire-related stimuli. Journal of Abnormal Child Psychology, 37, 1165-1176.

Green, M. W., \& Rogers, P. J. (1993). Selective attention to food and body shape words in dieters and restrained nondieters. International Journal of Eating Disorders, 14, 515-517.

Harrison, A., Sullivan, S., Tchanturia, K., \& Treasure, J. (2010). Emotional functioning in eating disorders: Attentional bias, emotion recognition and emotion. Psychological Medicine, 40, 1887-1897.

Heinz, A., Reimold, M., Wrase, J., Hermann, D., Croissant, B., Mundle, G., et al. (2005). Correlation of stable elevations in 
striatal mu-opioid receptor availability in detoxified alcoholic patients with alcohol craving: A positron emission tomography study using carbon 11-labeled carfentanil. Archives of General Psychiatry, 62, 57-64.

Hofmann, W., De Houwer, J., Perugini, M., Baeyens, F., \& Crombez, G. (2010). Evaluative conditioning in humans: A metaanalysis. Psychological Bulletin, 136, 390-421.

Houben, K., Roefs, A., \& Jansen, A. (2010). Guilty pleasures: Implicit preferences for high calorie food in restrained eating. Appetite, 55, 18-24.

Jansen, A., Huygens, K., \& Tenney, N. (1998). No evidence for a selective processing of subliminally presented body words in restrained eaters. International Journal of Eating Disorders, 24, $435-438$.

Johansson, L., Ghaderi, A., \& Andersson, G. (2005). Stroop interference for food- and body-related words: A meta-analysis. Eating Behaviors, 6, 271-281.

Jones-Chesters, M. H., Monsell, S., \& Cooper, P. J. (1998). The disorder-salient Stroop effect as a measure of psychopathology in eating disorders. International Journal of Eating Disorders, $24,68-82$.

Kemps, E., \& Tiggemann, M. (2009). Attentional bias for cravingrelated (chocolate) food cues. Experimental and Clinical Psychopharmacology, 17, 425-433.

Long, C. G., Hinton, C., \& Gillespie, N. K. (1994). Selective processing of food and body size words: Application of the Stroop test with obese restrained eaters, anorexics, and normals. International Journal of Eating Disorders, 15, 279-283.

Lundh, L. G., Wikstrom, J., Westerlund, J., \& Ost, L. G. (1999). Preattentive bias for emotional information in panic disorder with agoraphobia. Journal of Abnormal Psychology, 108, 222232.

MacLeod, C. (1991). Half a century of research on the Stroop effect: An integrative review. Psychological Bulletin, 109, 163203.

McNamara, T. P. (2005). Semantic priming: Perspective from memory and word recognition. New York: Psychology Press.

Mogg, K., \& Bradley, B. P. (2002). Selective processing of smoking-related cues in smokers: Manipulation of deprivation level and comparison of three measures of processing bias. Journal of Psychopharmacology, 16, 385-392.

Mogg, K., Bradley, B. P., Williams, R., \& Mathews, A. (1993). Subliminal processing of emotional information in anxiety and depression. Journal of Abnormal Psychology, 102, 304-311.

Orbell, S., \& Verplanken, B. (2010). The automatic component of habit in health behavior: Habit as cue-contingent automaticity. Health Psychology, 29, 374-383.

Papies, E. K., Stroebe, W., \& Aarts, H. (2009). Who likes it more? Restrained eaters' implicit attitudes towards food. Appetite, 53, 279-287.

Paunovi, N., Lundh, L. G., \& Ost, L. G. (2002). Attentional and memory bias for emotional information in crime victims with acute posttraumatic stress disorder (PTSD). Journal of Anxiety Disorders, 16, 675-692.

Perpina, C., Hemsley, D., Treasure, J., \& de Silva, P. (1993). Is the selective information processing of food and body words spe- cific to patients with eating disorders? International Journal of Eating Disorders, 14, 359-366.

Robinson, T. E., \& Berridge, K. C. (2001). Incentive-sensitization and addiction. Addiction, 96, 103-114.

Sackville, T., Schotte, D. E., Touyz, S. W., Griffiths, R., \& Beumont, P. J. (1998). Conscious and preconscious processing of food, body weight and shape, and emotion-related words in women with anorexia nervosa. International Journal of Eating Disorders, 23, 77-82.

Stroebe, W., Mensink, W., Aarts, H., Schut, H., \& Kruglanski, A. W. (2008). Why dieters fail: Testing the goal conflict model of eating. Journal of Experimental Social Psychology, 44, 26-36.

Tapper, K., Pothos, E. M., Fadardi, J. S., \& Ziori, E. (2008). Restraint, disinhibition and food-related processing bias. Appetite, 51, 335-338.

Thorpe, S. J., \& Salkovskis, P. M. (1997). Information processing in spider phobics: The Stroop colour naming task may indicate strategic but not automatic attentional bias. Behaviour Research and Therapy, 35, 131-144.

Tiffany, S. T. (1990). A cognitive model of drug urges and drug-use behavior: role of automatic and nonautomatic processes. Psychological Review, 97, 147-168.

Van den Bussche, E., Van den Noortgate, W., \& Reynvoet, B. (2009). Mechanisms of masked priming: A meta-analysis. Psychological Bulletin, 135, 452-477.

van der Made, F., Bloemers, J., Yassem, W. E., Kleiverda, G., Everaerd, W., van Ham, D., . . . Tuiten, A. (2009). The influence of testosterone combined with a PDE5-inhibitor on cognitive, affective, and physiological sexual functioning in women suffering from sexual dysfunction. The Journal of Sexual Medicine, 6, 777-790.

Vollstadt-Klein, S., Loeber, S., von der Goltz, C., Mann, K., \& Kiefer, F. (2009). Avoidance of alcohol-related stimuli increases during the early stage of abstinence in alcohol-dependent patients. Alcohol, 44, 458-463.

Walker, M. K., Ben-Tovim, D. I., Paddick, S., \& McNamara, J. (1995). Pictorial adaptation of Stroop measures of body-related concerns in eating disorders. International Journal of Eating Disorders, 17, 309-311.

Wiers, R. W., Teachman, B. A., \& De Houwer, J. (2007). Implicit cognitive processes in psychopathology: An introduction. Journal of Behavior Therapy and Experimental Psychiatry, 38, 95-104.

Williams, J. M. G., Mathews, A., \& MacLeod, C. (1996). The emotional Stroop task and psychopathology. Psychological Bulletin, 120, 3-24.

World Health Organization. (2010). Obesity. Retrieved from http:// www.who.int/infobase

Zetteler, J. I., Stollery, B. T., Weinstein, A. M., \& LingfordHughes, A. R. (2006). Attentional bias for alcohol-related information in adolescents with alcohol-dependent parents. Alcohol, 41, 426-430.

Received December 12, 2010

Revision received April 11, 2011 Accepted April 11, 2011 